\title{
A comparison of jamming behavior in systems composed of dimer- and ellipse-shaped particles
}

\author{
Carl F. Schreck ${ }^{1}$, Ning Xu², and Corey S. O'Hern ${ }^{3,1}$ \\ ${ }^{1}$ Department of Physics, Yale University, New Haven CT 06520-8120 \\ ${ }^{2}$ Department of Physics, University of Science and Technology, \\ Hefei 230026, China \\ ${ }^{3}$ Department of Mechanical Engineering, Yale University, \\ New Haven, CT 06520-8286
}

November 6, 2018

\begin{abstract}
We compare the structural and mechanical properties of static packings composed of frictionless convex (ellipses) and concave (rigid dimers) particles in two dimensions. We employ numerical simulations to generate static packings and measure the shear stress in response to applied simple shear strain as a function of the aspect ratio and amount of compression. We find that the behavior near jamming is significantly different for ellipses and dimers even though both shapes are roughly characterized by the aspect ratio and possess the same number of translational and rotational degrees of freedom per particle. For example, we find that ellipse packings are hypostatic (not isostatic as found for dimers), display novel power-law scaling of the static linear shear modulus and contact number with the amount of compression, and possess stressstrain relations that are qualitatively different from that for dimers. Thus, we observe that important macroscopic properties of static packings of anisotropic particles can depend on the microscale geometrical features of individual particles.
\end{abstract}

\section{Introduction}

Significant progress has been made in understanding the jamming transition that occurs in collections of frictionless spherical particles with purely repulsive short-range interactions, where systems transform from liquid- to solid-like states as a function of increasing packing fraction or decreasing applied shear stress $[1,2,3]$. Key findings include the power-law scaling of the static shear modulus with packing fraction above the onset of jamming $[4,5]$, the identification of a growing lengthscale as the system approaches the jamming transition [6], above which the system can be described as an elastic material [7], and an abundance of low-energy excitations in the density of vibrational modes [8]. Much of this behavior stems from the fact that frictionless, static packings of spherical particles are typically isostatic since they possess the minimal number of contacts per particle $z_{\text {iso }}=2 d$, where $d$ is the spatial dimension, required for mechanical stability [9].

However, less progress has been made in understanding the jamming transition in particulate systems composed of nonspherical particles, despite the fact that these systems display striking mechanical [10] and rheological [11] properties, and are more relevant for industrial applications and in nature. An important difference between static packings of frictionless spherical versus ellipsoidal particles is that the latter are typically hypostatic, not isostatic, with fewer contacts than required to constrain all of the translational and rotational degrees of freedom using straightforward counting 

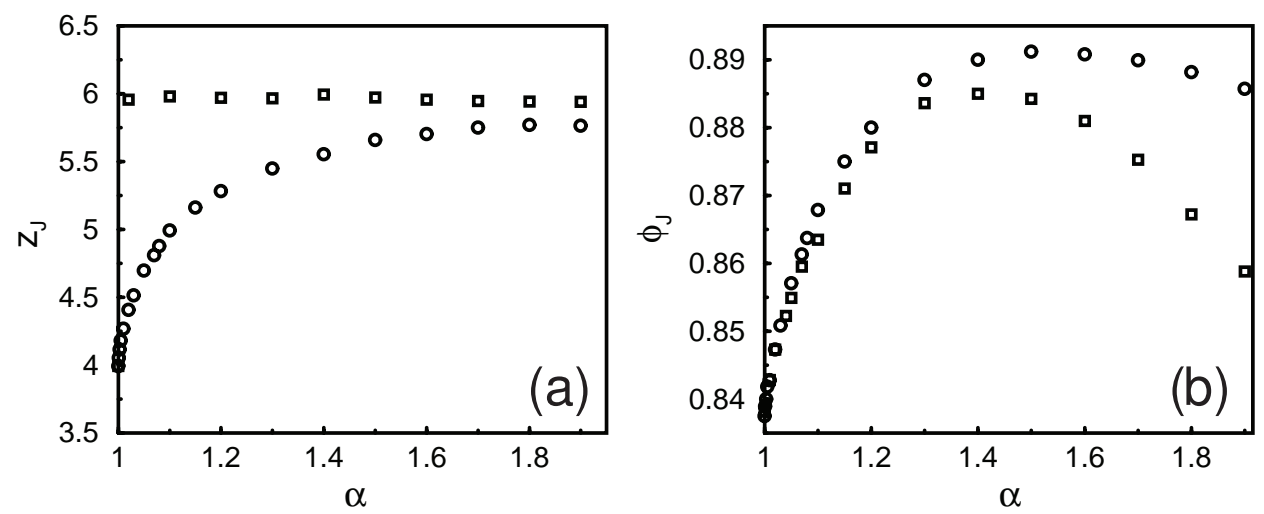

Figure 1: Ensemble averaged (a) contact number $z_{J}$ and (b) packing fraction $\phi_{J}$ at jamming as a function of aspect ratio $\alpha$ for dimers (squares) and ellipses (circles) for $N=480$ particles.

arguments $[12,13]$. Previous studies have found that for ellipse packings the contact number at jamming $z_{J}<z_{\text {iso }}=2 d_{f}$, where $d_{f}=3$ in two dimensions $(2 D)$, over a wide range of aspect ratios $\alpha$ [12]. We include similar results from our simulations of static ellipse packings in Fig. 1 (a). $z_{J}<z_{\text {iso }}$ for small aspect ratios, but slowly approaches a value $z_{J}^{*}$ that is only a few percent below the isostatic value as $\alpha$ increases. The packing fraction at jamming $\phi_{J}$ for ellipses, shown in panel (b), possesses a peak, which is only a few percent lower than the crystalline value for spherical particles, near $\alpha \sim 1.5[12,14]$.

\section{Motivation}

In this manuscript, we investigate the generality of these results for the behavior near jamming of frictionless, anisotropic particles by comparing the structural and mechanical properties of two classes of nonspherical shapes: convex (ellipses) and concave (rigid dimers) particles. We find that the behavior near jamming for rigid dimers differs significantly from that for ellipses even though both shapes are roughly characterized by the aspect ratio and possess the same number of translational and rotational degrees of freedom per particle.

We find several key differences between the structural and mechanical properties of static packings of dimers and ellipses. First, our simulations indicate that static packings of dimers are isostatic (not hypostatic as found for ellipses) with $z \simeq z_{\text {iso }}$ contacts per particle over the full range of aspect ratios studied as shown in Fig. 1 (a) ${ }^{1}$. Second, ellipse packings display novel power-law scaling of the static linear shear modulus $G$ and contact number $z-z_{J}$ with $\phi-\phi_{J}$ [15] - both scale linearly with $\phi-\phi_{J}$. In contrast, for dimer packings $G$ and $z-z_{J}$ scale as $\left(\phi-\phi_{J}\right)^{0.5}$, which is the same scaling found for static packings of spherical particles [4]. Third, we find that the shear stress-strain relations for packings of dimers and ellipses are qualitatively different. For example, at large compressions, the stress response (below the yield stress) to applied strain depends strongly on aspect ratio for ellipses, but it is nearly independent of aspect ratio for dimers. Also, at small compressions, dimer packings display nearly perfect plastic response in a region of strain where ellipse packings possess roughly linear response.

This manuscript is organized as follows. In Sec. 3 we describe the computational methods for generating static packings of ellipses and dimers and then applying quasistatic simple shear to measure the mechanical response. In Sec. 4, we present our results for the linear static shear

\footnotetext{
${ }^{1}$ It is still an open question which concave particle shapes yield isostatic packings, and which do not.
} 

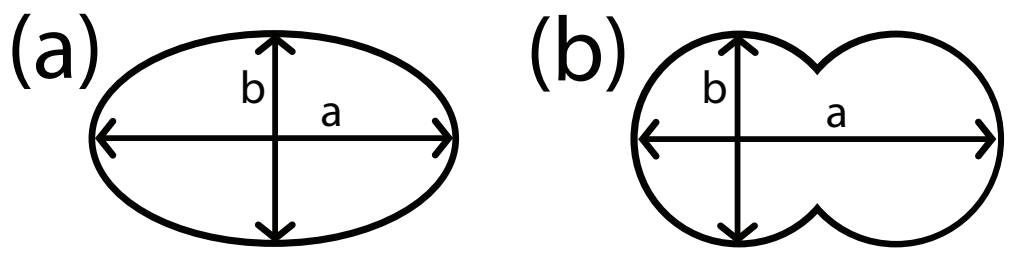

Figure 2: Definition of the aspect ratio $\alpha=a / b$ (ratio of the major to minor axes) for (a) ellipses and (b) dimers.

modulus, contact number, stress-strain relation, and particle rearrangement statistics. In Sec. 5, we discuss our conclusions and identify possible future research directions. We also include five appendices, which provide the details necessary for calculating the packing fraction for dimers, contact distance between ellipses, and forces, torques, and stress tensor for anisotropic particles [16].

\section{Computational methods}

We performed computational studies to measure the structural and mechanical properties of static packings of rigid dimers and ellipse-shaped particles in $2 D$. The particle shapes are shown in Fig. 2. The rigid dimers are formed by fusing identical disks together. We study aspect ratios $\alpha=a / b$ in the range $1 \leq \alpha \leq 2$, where $a$ and $b$ are the length of the major and minor axes, respectively. To inhibit crystallization, we focus on bidisperse mixtures of particles: $2 N / 3$ particles with minor axis $b$ and $N / 3$ larger particles with minor axis 1.4b. The particles are enclosed in square simulation cells with box length $L$ and periodic boundary conditions. System sizes were varied from $24 \leq N \leq 480$.

The particles interact via soft, pairwise, purely repulsive linear spring potentials. The total potential energy is therefore given by

$$
V=\sum_{i>j} V\left(\frac{r_{i j}}{\sigma_{i j}}\right)=\frac{\epsilon}{2} \sum_{i>j}\left(1-\frac{r_{i j}}{\sigma_{i j}}\right)^{2} \Theta\left(1-\frac{r_{i j}}{\sigma_{i j}}\right),
$$

where $\vec{r}_{i j}$ is the vector separation between the centers of particles $i$ and $j, \epsilon$ is the characteristic energy scale of the interaction, $\Theta(x)$ is the Heaviside function, and $\sigma_{i j}$ is the contact distance that in general depends on the orientation of particles $i$ and $j, \hat{\mu}_{i}$ and $\hat{\mu}_{j}$, and $\hat{r}_{i j}$.

Contact distance Determining the interactions between dimers is straightforward: one can identify overlaps between individual disks (monomers) on different dimers. Thus, the contact distance between disk $i$ on a given dimer and disk $j$ on a different dimer is $\sigma_{i j}=\left(b_{i}+b_{j}\right) / 2$, and the total potential energy can be obtained by summing up the contributions $V\left(r_{i j} / \sigma_{i j}\right)$ over all disk-disk interactions for disks on distinct dimers.

The contact distance $\sigma_{i j}$ between ellipses is more difficult to calculate than that for dimers. We define $\sigma_{i j}$ as the distance at which two ellipses will first come into contact when moved along their center-to-center direction while their orientations are held fixed. Fig. 3 illustrates how $\sigma_{i j}$ is measured for ellipses $i$ and $j$ with orientations $\hat{\mu}_{i}$ and $\hat{\mu}_{j}$ at separation $\vec{r}_{i j}$. We calculate the contact distances $\sigma_{i j}$ analytically in systems of bidisperse ellipses using the Perram-Wertheim formulation [17, 18, 19, 20]. Further details are provided in Appendix B.

Packing-generation algorithm We generate static, zero-pressure packings of bidisperse dimers and ellipses using a generalization of the compression/decompression method employed in our 
(a)

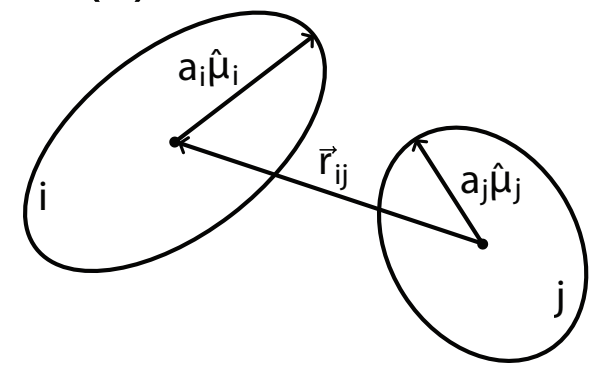

(b)

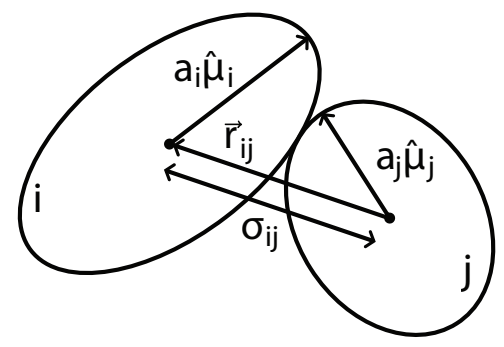

Figure 3: (a) Ellipses $i$ and $j$ with orientations $\hat{\mu}_{i}$ and $\hat{\mu}_{j}$ at center-to-center separation $\vec{r}_{i j}$. (b) The contact distance $\sigma_{i j}$ is obtained by identifying the point of contact when the two ellipses are brought together at fixed orientation.

previous studies of spherical particles [21, 22]. Representative packings of dimers and ellipses are shown in Fig. 4. We briefly outline the packing-generation procedure here for completeness.

We begin the packing-generation process by choosing random initial particle positions and orientations within the simulation cell at packing fraction $\phi_{0}=0.20$ (which is well below the minimum packing fraction at which frictionless packings of ellipses and dimers occur in $2 D$ for $1 \leq \alpha \leq 2)$. We successively increase or decrease the minor axes of the particles while maintaining the aspect ratio, with each compression or decompression step followed by conjugate gradient minimization [23] of the total energy (1). The system is decompressed when the total energy at a local minimum is nonzero-i.e., there are finite particle overlaps. If the potential energy of the system is zero and gaps exist between particles, the system is compressed. The increment by which the packing fraction $\phi$ is changed at each compression or decompression step is gradually decreased. The process is stopped when the total potential energy per particle $V / \epsilon N \ll 1$. Further details of the packing-generation algorithm are provided in Appendix C.

The packing fraction $\phi_{J}$, contact number $z_{J}$, and mechanical response are used to characterize each static packing. The packing-generation process is repeated at least 100 times at each $\alpha$ to generate configurational averages. Once the packings at jamming onset are generated, they can be successively compressed by small amounts $\Delta \phi$, followed by energy minimization at each step, to yield sets of configurations at fixed $\phi-\phi_{J}$.

Quasistatic simple shear To determine the mechanical properties of static packings of dimers and ellipses, we studied their response to quasistatic simple shear at fixed area. We first initialized the system with an unstrained packing at a given $\phi-\phi_{J}$ and successively applied to each particle $i$ small affine simple shear strain steps $\delta \gamma=\delta x / L$ along the $x$-direction with a gradient in the $y$-direction:

$$
x_{i} \rightarrow x_{i}+\delta \gamma y_{i}
$$

where $\vec{r}_{i}=\left(x_{i}, y_{i}\right)$ is the location of the center of mass of particle $i$. To be consistent with simple shear, at each strain step, the angle $\theta_{i}=\cos ^{-1}\left(\hat{\mu}_{i} \cdot \hat{x}\right)$ that particle $i$ makes with the $x$-axis was also rotated:

$$
\theta_{i} \rightarrow \cot ^{-1}\left(\cot \theta_{i}+\delta \gamma\right)
$$



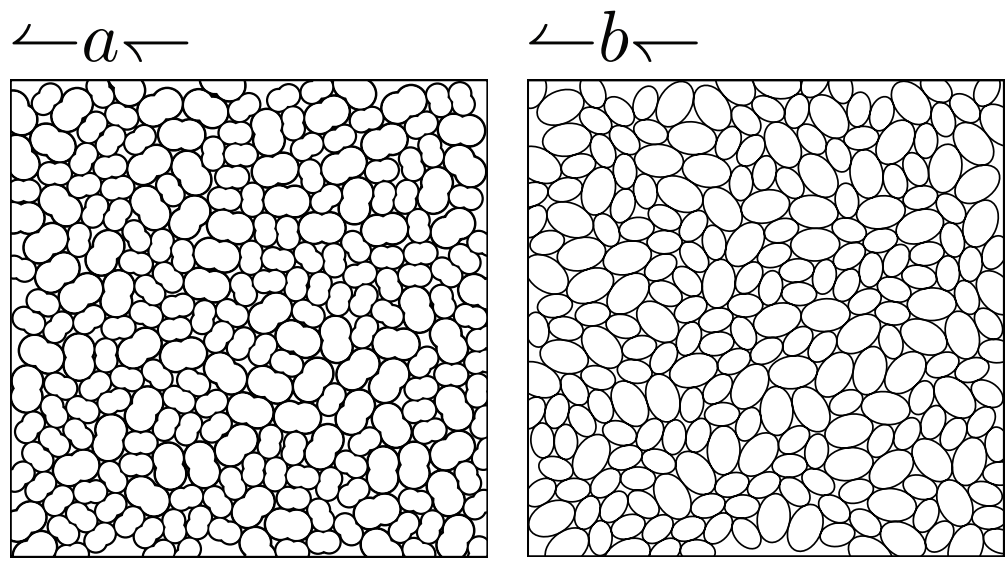

Figure 4: Snapshots of static packings of $N=240$ bidisperse (a) rigid dimers and (b) ellipses with $\alpha=1.5$.

Each shear strain step was followed by conjugate gradient energy minimization using Lees-Edwards (shear periodic) boundary conditions [24]. For most studies, $\delta \gamma=10^{-3}$ with accumulated strains $\gamma_{t}=1$. We verified that smaller strain steps yielded similar results. During the quasistatic shear, we measured the shear stress (in units of $\epsilon / b$ ), contact number, and statistics of particle rearrangement events. These measurements are described in the following Sec. 4. Details of the stress calculations are provided in Appendices D and E.

\section{Results and discussion}

We present several measurements of the structural and mechanical properties of ellipse and dimer packings as a function of aspect ratio and compression $\phi-\phi_{J}$ including the contact number, shear modulus, yield stress, and other features of the stress-strain relations.

Contact number at jamming The contact number is defined by $z=N_{c} /\left(N-N_{r}\right)$, where $N_{c}$ is the number of contacts (interparticle overlaps) in the packing. $N_{r}$ is the number of rattler particles with fewer than three contacts. The contact network is found by identifying all interparticle contacts, and then recursively removing rattler particles until there are none remaining in the packing.

In Fig. 1 (a) we show results for the contact number $z_{J}$ at jamming for ellipse and dimer packings. We find that ellipse packings are hypostatic with $z_{J}<2 d_{f}$ over the range of aspect ratio $1 \leq \alpha \leq 2$, while dimer packings are isostatic with $z_{J} \simeq 2 d_{f}$ over the same range of $\alpha$. We showed previously $[15,20]$ that hypostatic packings of ellipsoidal particles possess vibrational modes that are quartically (not quadratically) stabilized with the number of quartic modes determined by the deviation from isostaticity, $z_{\text {iso }}-z_{J}$. In contrast, all vibrational modes for dimer packings are quadratically stabilized since they are isostatic.

Power-law scaling of linear shear modulus and contact number One of the hallmarks of the jamming transition in packings of spherical particles is the power-law scaling of the static linear shear modulus $G$ and contact number $z-z_{J}$ with $\phi-\phi_{J}$. Both scale as $\left(\phi-\phi_{J}\right)^{0.5}$ for linear repulsive springs, which suggests that the contact number scaling controls the behavior of the linear shear modulus [4]. In Fig. 5, we plot $G$ as a function of $\phi-\phi_{J}$ for dimers (filled symbols) 


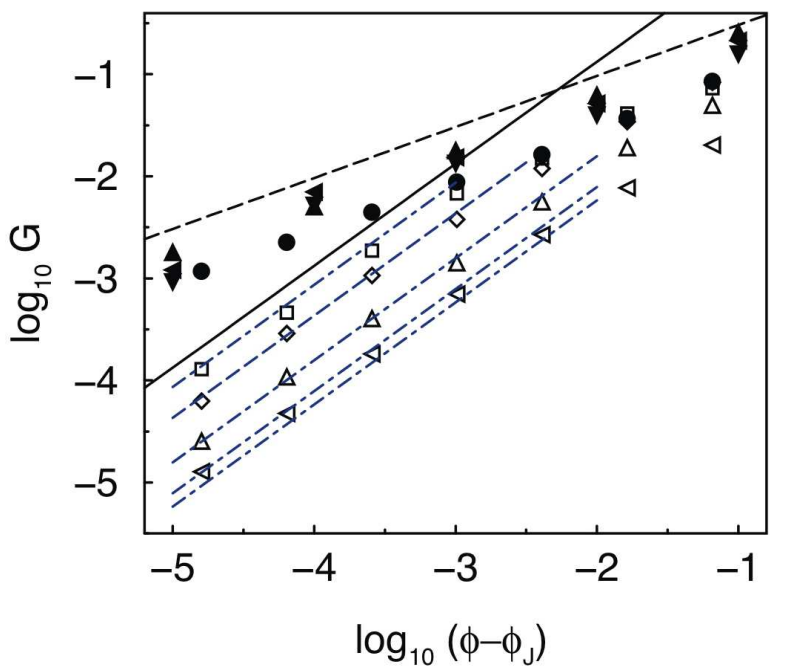

Figure 5: Static shear modulus $G$ versus $\phi-\phi_{J}$ for $N=480$ ellipses (open symbols) and 240 dimers (filled symbols) at $\alpha=1.0$ (circles), 1.002 (squares), 1.01 (diamonds), 1.05 (upward triangles), 1.1 (leftward triangles), 1.5 (downward triangles), and 2.0 (rightward triangles). The solid (dashed) line has slope $1(0.5)$. The dot-dashed lines have the form $G=0.6\left(\phi-\phi_{J}\right) /(\alpha-1)^{0.44}$.

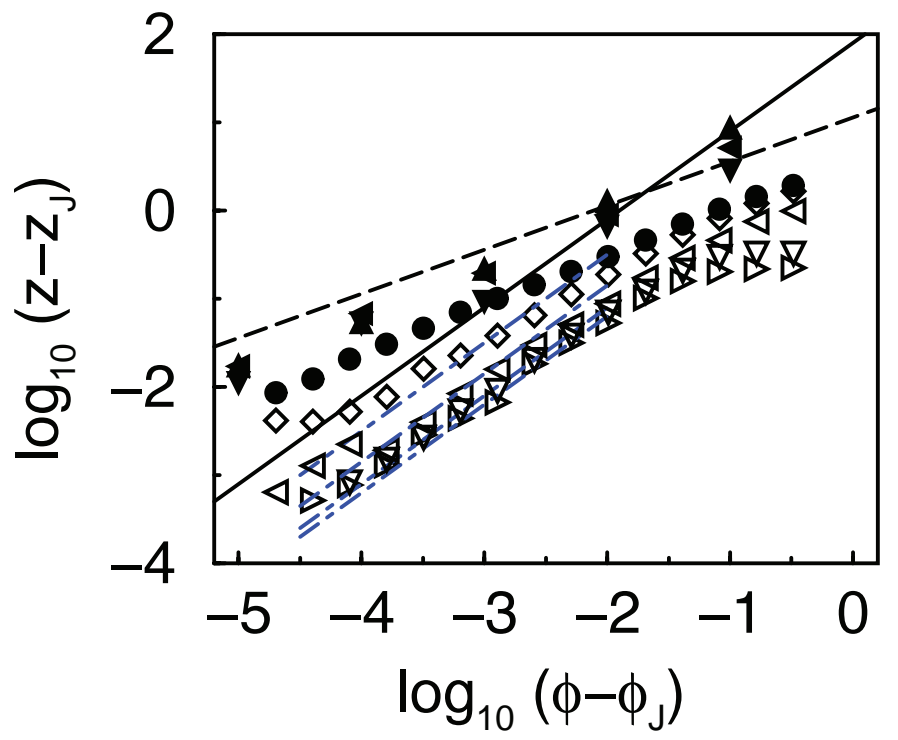

Figure 6: Deviation in the contact number $z$ from the value at jamming $z_{J}$ versus $\phi-\phi_{J}$ for $N=480$ ellipses (open symbols) and 240 dimers (filled symbols) at $\alpha=1.0$ (circles), 1.002 (squares), 1.01 (diamonds), 1.05 (upward triangles), 1.1 (leftward triangles), 1.5 (downward triangles), and 2.0 (rightward triangles). The solid (dashed) line has slope $1(0.5)$. The dot-dashed lines have the form $z-z_{J}=6.3\left(\phi-\phi_{J}\right) /(\alpha-1)^{0.35}$. 

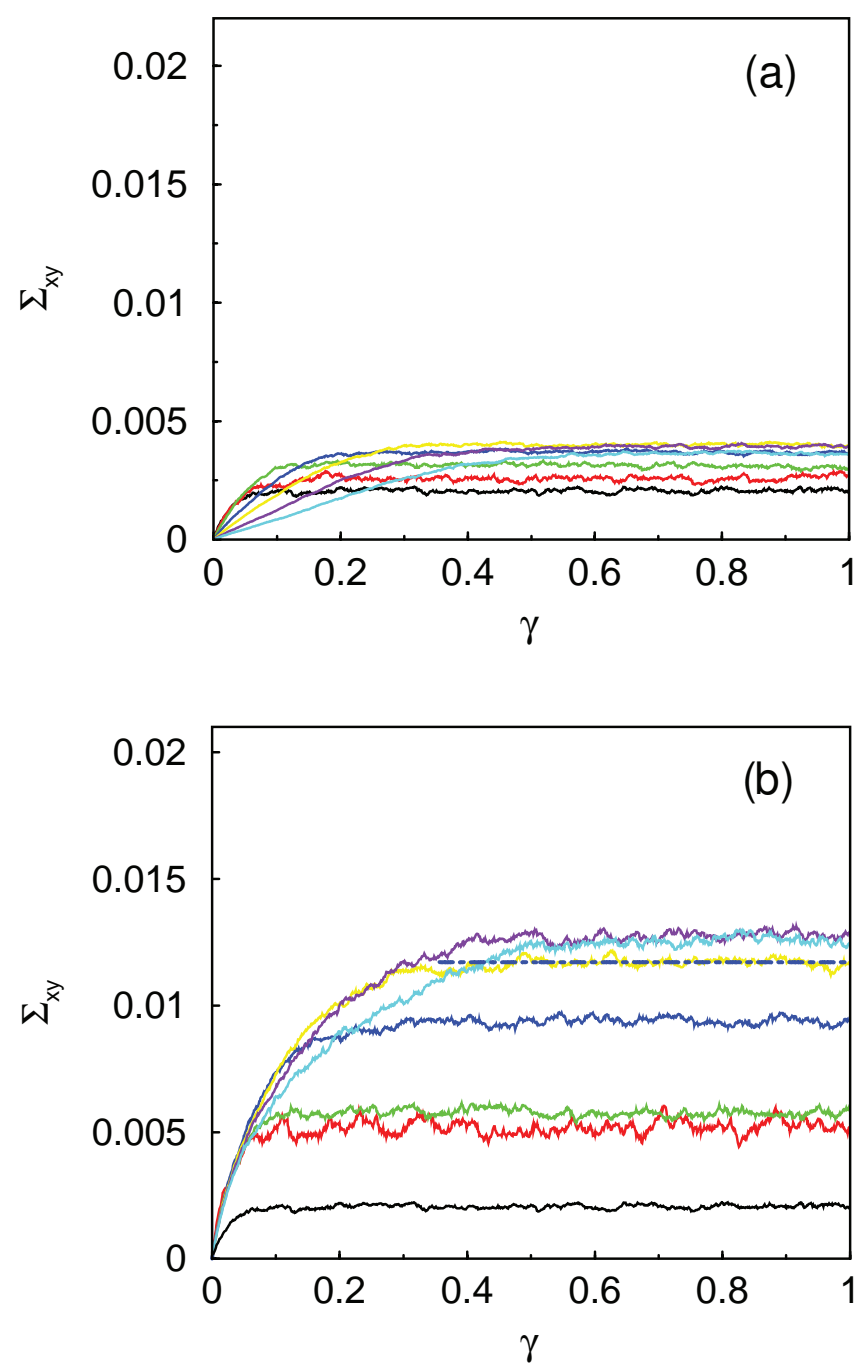

Figure 7: Shear stress $\Sigma_{x y}$ versus shear strain $\gamma$ for packings of (a) ellipses and (b) dimers at $\phi-\phi_{J}=10^{-1}$ for several aspect ratios $\alpha=1.0$ (black), 1.05 (red), 1.1 (green), 1.2 (blue), 1.3 (yellow), 1.4 (violet), and 1.5 (cyan). The dashed horizontal line in (b) indicates the yield stress for dimer packings at $\alpha=1.3$.

and ellipses (open symbols) over a range of aspect ratios. We again find power-law scaling near jamming,

$$
G=G_{0}(\alpha)\left(\phi-\phi_{J}\right)^{\beta},
$$

where $\beta=0.5$ and $G_{0}$ is weakly dependent on $\alpha$ for dimers. In contrast, $\beta=1$ for sufficiently small $\phi-\phi_{J}$ and $G_{0}(\alpha) \sim(\alpha-1)^{-0.44 \pm 0.03}$ for ellipses. The power-law scaling is stronger for ellipse packings, and thus the ratio of the shear moduli $G_{\text {ellipse }} / G_{\text {dimer }} \rightarrow 0$ in the limit $\phi \rightarrow \phi_{J}$ for all $\alpha$. This implies that ellipse packings are much more susceptible to shear in the linear response regime.

For jammed packings of spherical particles with linear spring interactions both $G$ and $z-z_{J}$ scale as $\left(\phi-\phi_{J}\right)^{0.5}$. We find similar behavior, $G \sim z-z_{J}$, for dimer packings as a shown in Fig. 6 . For ellipse packings, we find

$$
z-z_{J}=z_{0}(\alpha)\left(\phi-\phi_{J}\right)^{\beta}
$$

where $z_{0}(\alpha) \sim(\alpha-1)^{-0.35 \pm 0.1}$ and $\beta=1$ for sufficiently small $\phi-\phi_{J}$. Thus, $G$ and $z-z_{J}$ have the 

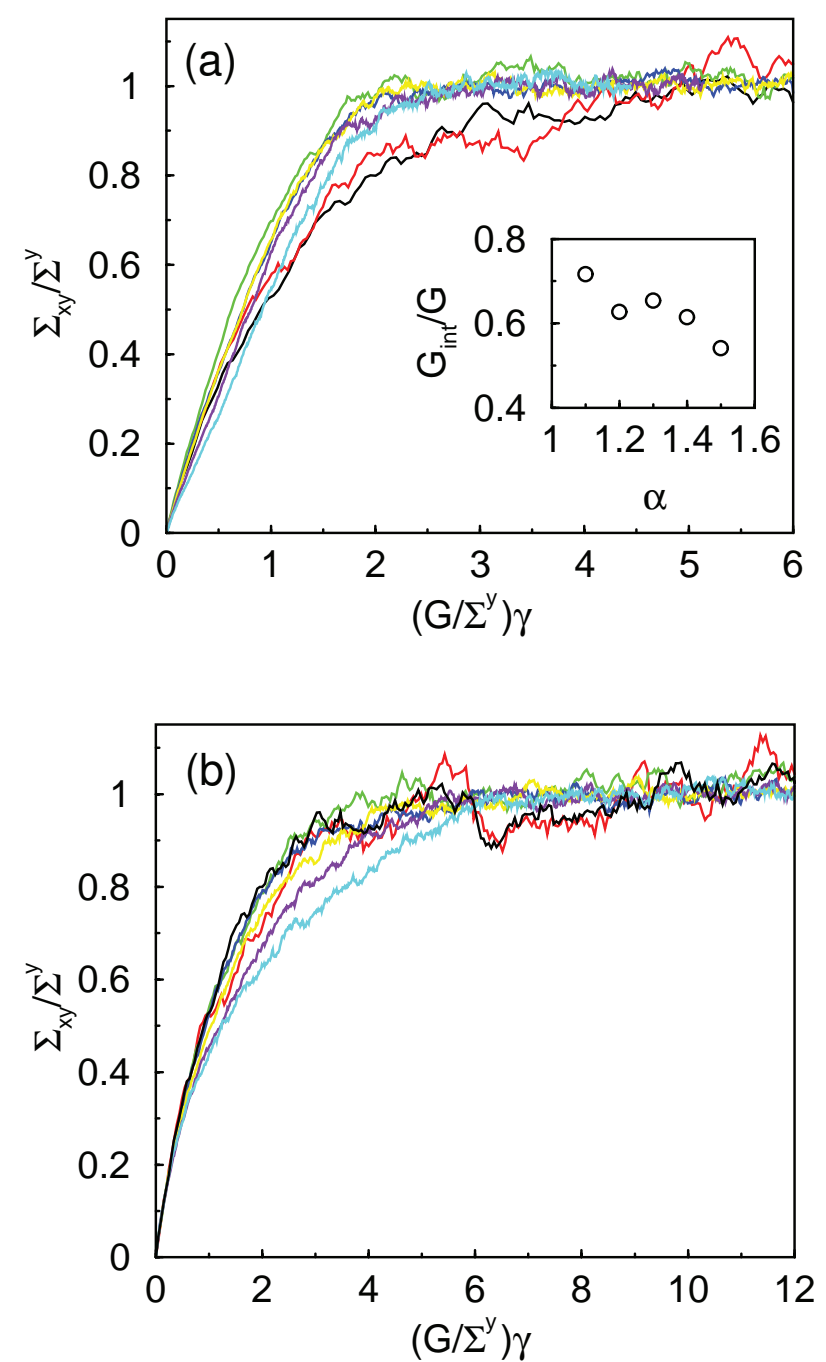

Figure 8: Same stress-strain relations in Fig. 7 for (a) ellipses and (b) dimers except the shear stress and strain have been scaled by $\Sigma^{y}$ and $\Sigma^{y} / G$, respectively. The inset to panel (a) gives the average value of the slope $\left(G_{\text {int }} / G\right)$ of the scaled stress-strain relation for shear stresses $\Sigma_{x y}<\Sigma^{y}$ at aspect ratios $\alpha \geq 1.1$.

same power-law scaling with $\phi-\phi_{J}$ even for hypostatic packings. We argued previously that the novel power-law scaling exponent for $G$ and $z-z_{J}$ in ellipse packings originates from the quartically stabilized vibrational modes [15].

Stress-strain relations The full stress-strain behavior for ellipse and dimer packings is complex; it is qualitatively different for ellipses and dimers and depends nontrivially on $\phi-\phi_{J}$ and aspect ratio. In Figs. 7 and 9, we show the shear stress $\Sigma_{x y}$ versus strain $\gamma$ for $\phi-\phi_{J}=10^{-1}$ and $10^{-3}$. For ellipses at $\phi-\phi_{J}=10^{-1}$ (Fig. 7 (a)), the shear stress is roughly linear with strain until the shear stress plateaus at the yield stress, $\Sigma^{y}=\Sigma_{x y}(\gamma \rightarrow \infty)$, which only weakly depends on aspect ratio and is at least a factor of 2 smaller than that for dimers (c.f. Fig. 11 (a)). We can achieve an approximate collapse of the stress-strain data for ellipses at $\phi-\phi_{J}=10^{-1}$ for $\alpha \geq 1.1$ by scaling the shear stress by $\Sigma_{y}$ and strain by $\Sigma_{y} / G$ as shown in Fig. 8 (a). The inset to Fig. 8 (a) shows that the average shear modulus defined over the wide range $0 \leq \Sigma_{x y} \leq \Sigma^{y}$ is comparable to the 

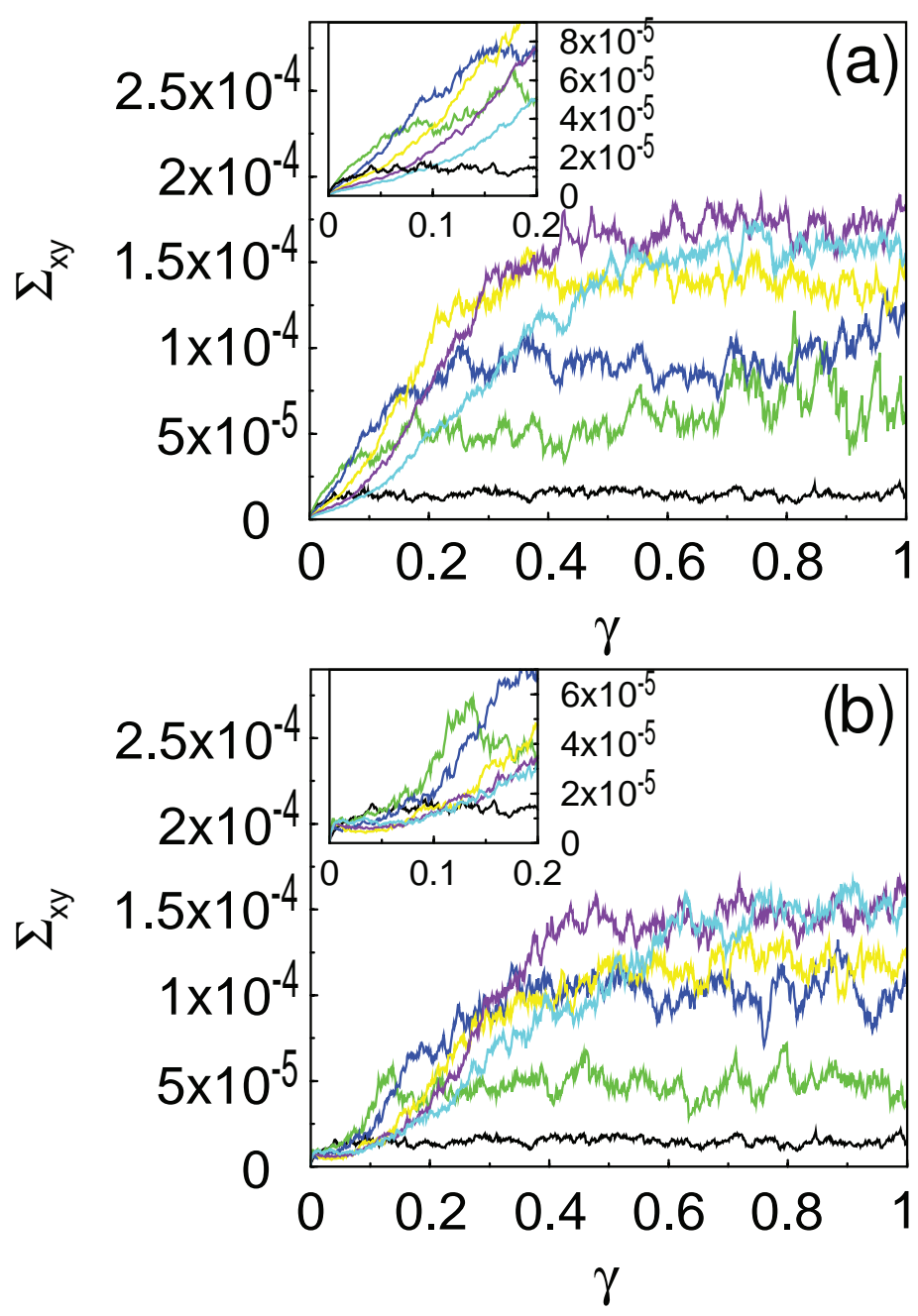

Figure 9: Shear stress $\Sigma_{x y}$ versus shear strain $\gamma$ for packings of (a) ellipses and (b) dimers at $\phi-\phi_{J}=10^{-3}$ for several aspect ratios $\alpha=1.0$ (black), 1.1 (green), 1.2 (blue), 1.3 (yellow), 1.4 (violet), and 1.5 (cyan). The insets show the same data as in the main plots, except over a smaller range of $\gamma$.

linear response value, $G$, at small strains (c.f. Fig. 5) for $\alpha \geq 1.1$.

The behavior of the stress-strain curves for dimers at $\phi-\phi_{J}=10^{-1}$ is qualitatively different from that for ellipses as shown in Fig. 7 (b). In particular, the approach of the shear stress to the yield stress plateau has significant curvature similar to the behavior found for sheared packings of spherical particles [25]. The scaled stress-strain curve in Fig. 8 (b) emphasizes that dimer packings further strain soften as the aspect ratio increases.

Fig. 9 shows the stress-strain behavior for dimers and ellipses much closer to the jamming transition at $\phi-\phi_{J}=10^{-3}$. At such small compressions, ellipse packings (panel (a)) no longer possess such robust, sustained linear response over the range $0 \leq \Sigma_{x y} \leq \Sigma^{y}$. Instead, the shear stress is first roughly linear with slope $\sim G$, but then stiffens on approach to the yield stress. However, the most striking feature of the stress-strain curves at $\phi-\phi_{J}=10^{-3}$ is the nearly perfect plastic response (flat shear stress versus strain) for dimer packings (panel (b)) with $\alpha \geq 1.1$. The plastic regime extends for strains from the end of the linear response regime to $\gamma_{p} \approx 0.1$. For $\gamma>\gamma_{p}$, the shear stress grows rapidly as it approaches the yield stress, which is similar to the 

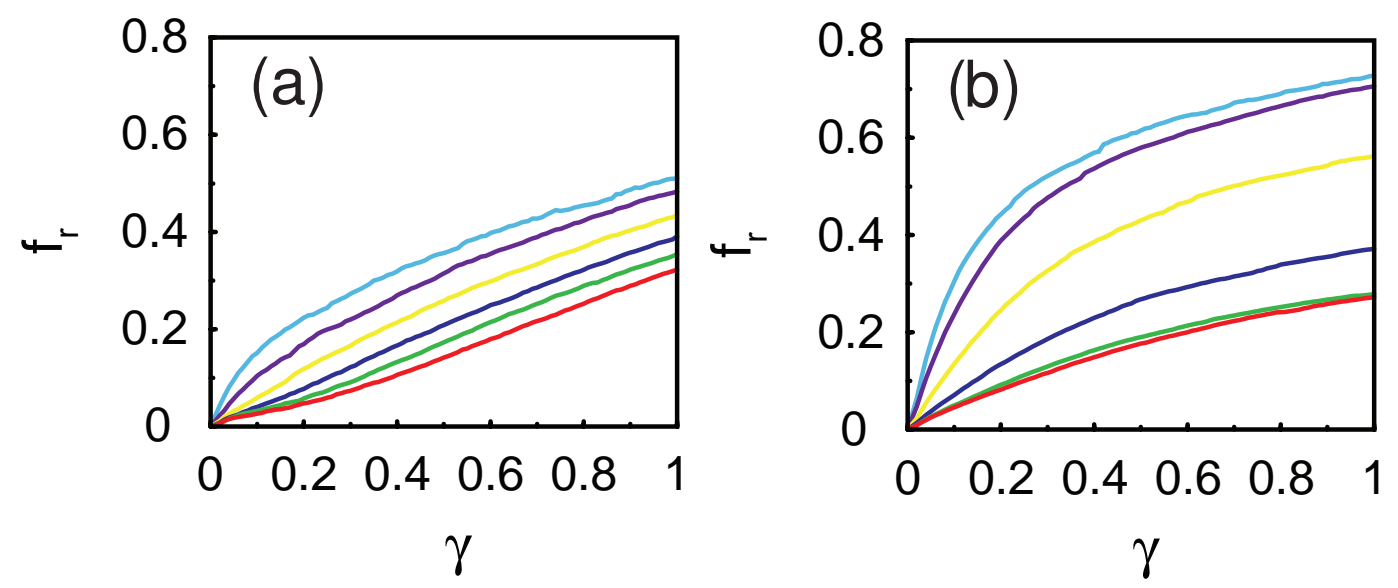

Figure 10: Fraction $f_{r}$ of the particle contacts at strain $\gamma$ that differ from those at $\gamma=0$ for (a) ellipses and (b) dimers at $\alpha=1.05$ (red), 1.1 (green), 1.2 (blue), 1.3 (yellow), 1.4 (violet), and 1.5 (cyan).

behavior in Fig. 7 (b) at $\phi-\phi_{J}=10^{-1}$.

As demonstrated in the inset to Fig. 9 (a), ellipse packings do not possess this nearly plastic response. In the regime $\gamma<\gamma_{p}$, the shear stress is roughly linear with a shear modulus comparable to $G$. In Fig. 10, we plot the fraction of particle contacts at strain $\gamma$ that differ from those at $\gamma=0$. We find that the plastic behavior in dimer packings is accompanied by a large increase in the number of particle rearrangement events (changes in the contact network) over the strain interval $0 \leq \gamma \leq \gamma_{p}$. Note that the largest fraction (and rate of increase over $0 \leq \gamma \leq \gamma_{p}$ ) of particle rearrangements occurs for dimer packings with $\alpha=1.5$, which possess the most pronounced plastic response. Further work is required to elucidate the particle-scale motions that cause this plastic response.

We have also calculated the nematic order parameter, $S=\left\langle\cos \left[2\left(\theta-\theta_{0}\right)\right]\right\rangle$, where $\theta_{0}$ is average orientation of the particles, as a function of shear strain. We find that the nematic order increases roughly linearly with $\gamma$ up to strains of $0.2-0.3$ in both sheared dimer and ellipse packings. Thus, it is possible that nematic order leads to qualitatively different effects in dimer and ellipse packings since dimers display plastic response, while ellipses do not.

In Fig. 11, we show the yield stress $\Sigma^{y}$ for dimers and ellipses as a function of aspect ratio at (a) $\phi-\phi_{J}=10^{-1}$, (b) $10^{-2}$, and (c) $10^{-3}$. We find that the yield stress increases with aspect ratio, which acts as an effective friction coefficient [26]. However, $\Sigma^{y}$ begins to level off near $\alpha^{*} \sim 1.4$, which is likely related to a maximum in the nematic order near $\alpha^{*}$. In contrast to the behavior at small shear strains, the yield stress for dimers and ellipses becomes nearly identical near jamming at $\phi-\phi_{J}=10^{-3}$. Thus, measurements of the jamming packing fraction $\phi_{J}$ and yield stress $\Sigma^{y}$ are relatively insensitive to microscale geometrical features of individual particles.

\section{$5 \quad$ Future Directions}

These studies of the structural and mechanical properties of dimer and ellipse packings raise a number of interesting questions that will likely spur new research activity in this area. First, there are many features of jammed ellipse and dimer packings that are different. Most notably, ellipse packings are hypostatic while dimer packings are isostatic, which gives rise to novel power-law scaling of the structural and mechanical properties near jamming. Thus, we have shown that 

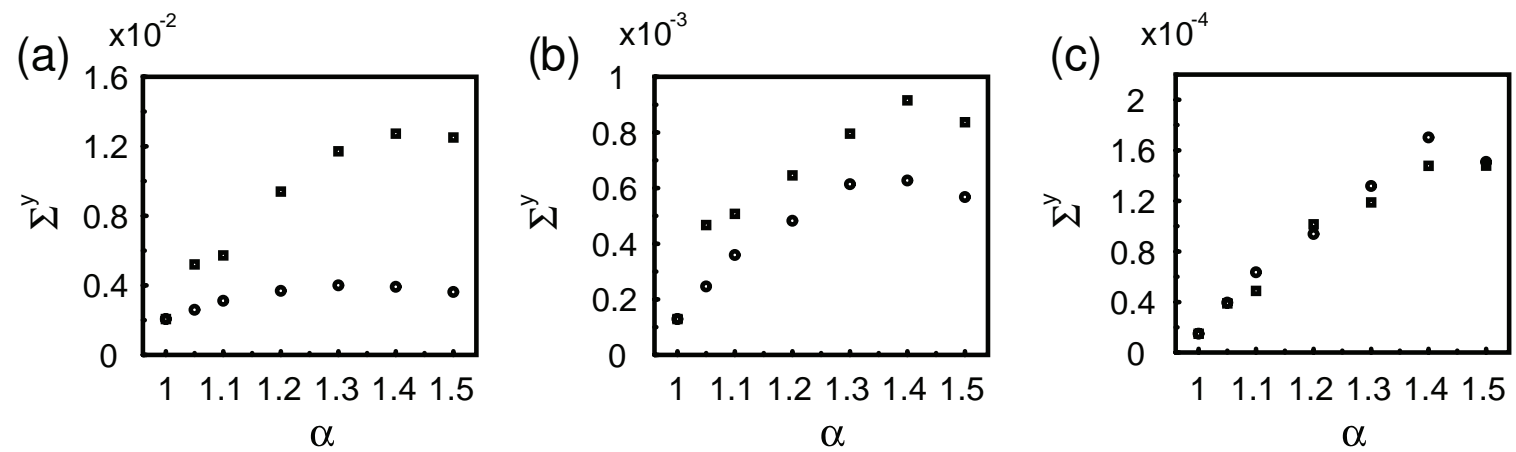

Figure 11: Yield stress $\Sigma^{y}$ as a function of aspect ratio $\alpha$ at (a) $\phi-\phi_{J}=10^{-1}$, (b) $10^{-2}$, and (c) $10^{-3}$ for ellipses (circles) and dimers (squares).

the macroscopic jamming behavior of anisotropic particles depends sensitively on the microscale geometrical features of individual particles.

However, the dependence on aspect ratio of the jamming packing fraction and yield stress, which strongly affect the glass transition in thermalized systems [27], is relatively insensitive to whether the packings are composed of dimers or ellipses. Thus, it is not clear a priori which structural, mechanical, and dynamical properties are sensitive to microscale particle properties. Thus, we encourage reinvigorated studies of atomic, colloidal, and granular systems to determine under what circumstances geometrical features of individual particles play an important role in jamming behavior and glassy dynamics.

\section{Acknowledgments}

This work was supported by the National Science Foundation under Grant Nos. CAREERDMR0448838 and DMS0835742. The authors are grateful to B. Chakraborty, A. Donev, E. Dufresne, M. Mailman, K. Schweizer, and S. Torquato for helpful discussions. We also thank the Yale High Performance Computing Center for providing the required computing resources.

\section{A Packing fraction}

In our numerical simulations, we consider bidisperse mixtures of dimers and ellipses in which onethird $\left(N_{l}=1 / 3\right)$ of the particles are large (with a minor axis 1.4 times that of the smaller particles, i.e. $\left.b_{l}=1.4 b_{s}\right)$ and two-thirds $\left(N_{s}=2 / 3\right)$ of the particles are small. When calculating the packing fraction for rigid dimers (fused disks), we do not double count the overlapping region. Thus, we define the packing fraction for dimers in $2 D$ as

$$
\phi_{\text {dimer }}=2 N_{s} \pi\left(\frac{b_{s}}{L}\right)^{2}\left(1+\frac{N_{l}}{N_{s}}\left(\frac{b_{l}}{b_{s}}\right)^{2}\right)\left(1-\frac{1}{\pi}\left[\cos ^{-1}(\alpha-1)+(\alpha-1) \sqrt{\alpha(2-\alpha)}\right]\right) .
$$

For ellipses

$$
\phi_{\text {ellipse }}=N_{s} \pi \alpha\left(\frac{b_{s}}{L}\right)^{2}\left(1+\frac{N_{l}}{N_{s}}\left(\frac{b_{l}}{b_{s}}\right)^{2}\right)
$$




\section{B Contact distance}

The Perram and Wertheim formulation for calculating the contact distance $\sigma_{i j}$ between ellipses $i$ and $j$ with orientations $\hat{\mu}_{i}$ and $\hat{\mu}_{j}$ and center-to-center direction $\hat{r}_{i j}$ involves the following minimization procedure [18]:

$$
\sigma_{i j}=\min _{\lambda} \sigma(\lambda)=\min _{\lambda} \frac{\sigma_{0}(\lambda)}{\sqrt{1-\frac{\chi(\lambda)}{2} \sum_{ \pm} \frac{\left(\beta(\lambda) \hat{r}_{i j} \cdot \hat{\mu}_{i} \pm \beta(\lambda)^{-1} \hat{r}_{i j} \cdot \hat{\mu}_{j}\right)^{2}}{1 \pm \chi(\lambda) \hat{\mu}_{i} \cdot \hat{\mu}_{j}}}}
$$

where

$$
\begin{gathered}
\sigma_{0}(\lambda)=\frac{1}{2}\left(\frac{b_{i}^{2}}{\lambda}+\frac{b_{j}^{2}}{1-\lambda}\right)^{1 / 2}, \\
\chi(\lambda)=\left(\frac{\left(a_{i}^{2}-b_{i}^{2}\right)\left(a_{j}^{2}-b_{j}^{2}\right)}{\left(a_{j}^{2}+\frac{1-\lambda}{\lambda} b_{i}^{2}\right)\left(a_{i}^{2}+\frac{\lambda}{1-\lambda} b_{j}^{2}\right)}\right)^{1 / 2},
\end{gathered}
$$

and

$$
\beta(\lambda)=\left(\frac{\left(a_{i}^{2}-b_{i}^{2}\right)\left(a_{j}^{2}+\frac{1-\lambda}{\lambda} b_{i}^{2}\right)}{\left(a_{j}^{2}-b_{j}^{2}\right)\left(a_{i}^{2}+\frac{\lambda}{1-\lambda} b_{j}^{2}\right)}\right)^{1 / 4} .
$$

Determining $\lambda_{\min }$ that minimizes $\sigma(\lambda)$ (Eq. 8) involves solving for the roots of a quartic polynomial in $\lambda$ for $2 D$ bidisperse systems [20]. The polynomials can be expressed analytically in terms of $\hat{\mu}_{i}$, $\hat{\mu}_{j}, \hat{r}_{i j}$, and the major and minor axes of particles $i$ and $j$, and then solved using Newton's method.

\section{Packing-generation algorithm}

In Sec. 3, we outlined our procedure to generate static packings of dimers and ellipses. Here, we provide some of the numerical parameters involved in the simulations. For the energy minimization, we employ the conjugate gradient technique [23], where the particles are treated as massless. The two stopping criteria for the energy minimization are $V_{t}-V_{t-1}<V_{\mathrm{tol}}=10^{-12}$ and $V_{t}<V_{\min }=$ $10^{-12}$, where $V_{t}$ is the potential energy per particle at iteration $t$, and the target potential energy per particle of a static packing is $V_{\mathrm{tol}}<V / N<2 V_{\mathrm{tol}}$. For the first compression or decompression step we use the packing-fraction increment $\Delta \phi=10^{-3}$. Each time the procedure switches from expansion to contraction or vice versa, $\Delta \phi$ is reduced by a factor of 2 . Using the packing generation procedure with these parameters, we are able to locate the jamming threshold in packing fraction $\phi_{J}$ to within $10^{-6}$ for each static packing.

\section{Calculation of forces and torques}

In this appendix, we provide specific details for calculating the interparticle forces and torques for dimers and ellipses, which are required to perform energy minimization and evaluate the shear stress. The forces and torques can be obtained from the interaction potential (Eq. 1) using generalizations of $\vec{F}_{i j}=d V / d \vec{r}_{i j}$, where $\vec{F}_{i j}$ is the force on particle $i$ due to particle $j$. 

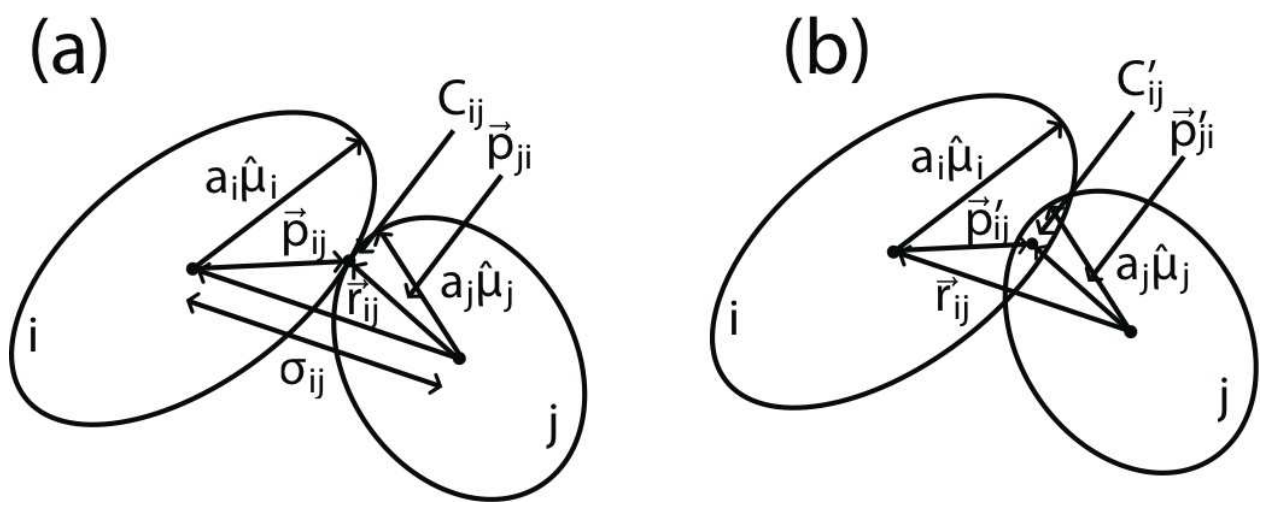

Figure 12: Definition of the point of contact $C_{i j}$ for ellipses $i$ and $j$ that are (a) 'just touching' and (b) overlapped. $\vec{p}_{i j}$ is location of the point of contact relative to the center of mass of ellipse $i$. In the overlapped case, the effective point of contact $C_{i j}^{\prime}$ is given by $\vec{p}_{i j}^{\prime}=\vec{p}_{i j}\left(r_{i j} / \sigma_{i j}\right)$.

Dimers For dimers, the interaction force on monomer $k_{i}$ belonging to dimer $i$ from monomer $k_{j}$ belonging to a distinct dimer $j$ is

$$
\vec{F}_{k_{i}, k_{j}}=\frac{d V}{d \vec{r}_{k_{i}, k_{j}}} .
$$

The total force on dimer $i$ is obtained by summing over all monomers $k_{i}$ belonging to dimer $i$, all dimers $j$ different from $i$, and all monomers $k_{j}$ belonging to dimer $j$ :

$$
\vec{F}_{i}=\sum_{k_{i}} \sum_{j} \sum_{k_{j}} \vec{F}_{k_{i}, k_{j}}
$$

The torque on dimer $i$ arising from an interaction between monomer $k_{i}$ on dimer $i$ and monomer $k_{j}$ belonging to dimer $j$ is given by

$$
\vec{T}_{k_{i}, k_{j}}=\vec{r}_{k_{i}} \times \vec{F}_{k_{i}, k_{j}}
$$

where $\vec{r}_{k_{i}}=d_{i}\left(\cos \theta_{i} \hat{x}-\sin \theta_{i} \hat{y}\right)$ is the vector from the center of dimer $i$ to the center of monomer $k_{i}, d_{i}=b_{i}(\alpha-1) / 2$, and $\theta_{i}$ gives the orientation of dimer $i$. The total torque on dimer $i, \vec{T}_{i}$, is obtained by summing $\vec{T}_{k_{i}, k_{j}}$ over all monomers $k_{i}$ on dimer $i$, all dimers $j$ distinct from $i$, and all monomers $k_{j}$ on dimer $j$.

Ellipses For ellipses $i$ and $j$, the interparticle force depends explicitly on how the contact distance $\sigma_{i j}$ varies with the vector separation $\vec{r}_{i j}$ :

$$
F_{\xi i j}=-\frac{\partial V}{\partial r_{i j}}\left(\frac{\xi_{i j}}{r_{i j}}-\frac{r_{i j}}{\sigma_{i j}} \frac{\partial \sigma_{i j}}{\partial \xi_{i j}}\right),
$$

where $\xi=x, y$. To calculate the torque, one must specify the point of contact. For 'just touching' ellipses $i$ and $j$ are in contact at only one point, as shown in Fig. 12 (a), the location $\vec{p}_{i j}$ of the 
point of contact $C_{i j}$ (relative to the center of mass of ellipse $i$ ) is unambiguous and given by

$$
\begin{aligned}
\vec{p}_{i j} & =p_{i j}^{0}\left(\cos \left(\psi_{i j}+\theta_{i}\right) \hat{x}+\sin \left(\psi_{i j}+\theta_{i}\right) \hat{y}\right) \\
p_{i j}^{0} & =\frac{1}{2 \sqrt{\left(\frac{\cos \psi_{i j}}{a_{i}}\right)^{2}+\left(\frac{\sin \psi_{i j}}{b_{i}}\right)^{2}}} \\
\tan \psi_{i j} & =\frac{1}{\alpha^{2}} \frac{\tan \left(\Theta_{i j}-\theta_{i}\right)-\sigma_{i j}^{-1} \frac{\partial \sigma_{i j}}{\partial \beta_{i j}}}{1+\sigma_{i j}^{-1} \tan \left(\Theta_{i j}-\theta_{i}\right) \frac{\partial \sigma_{i j}}{\partial \Theta_{i j}}},
\end{aligned}
$$

where $\cos \beta_{i j}=\hat{\mu}_{i} \cdot \hat{r}_{i j}, \cos \Theta_{i j}=\hat{x}_{i} \cdot \hat{r}_{i j}$. The torque $T_{i j}$ on ellipse $i$ from $j$ is then

$$
T_{i j}=p_{x i j} F_{y i j}-p_{y i j} F_{x i j} .
$$

As shown in Fig. 12 (b), upon compression, ellipses are no longer 'just touching', and thus Eq. (16) for the point of contact $C_{i j}$ is no longer exact. In this case, we scale $\vec{p}_{i j}$ by $r_{i j} / \sigma_{i j}$, which yields an effective point of contact $C_{i j}^{\prime}$ that is within the overlap region of the two ellipses.

\section{E Calculation of shear stress}

For systems composed of spherical particles, the correct form for the stress tensor $\hat{\Sigma}_{\alpha \beta}$ in $2 D$, where $\alpha, \beta=x, y$, is the virial expression [24]:

$$
\hat{\Sigma}_{\alpha \beta}^{V}=\frac{1}{2 L^{2}} \sum_{i>j=1}^{N}\left(F_{i j \alpha} r_{i j \beta}+F_{i j \beta} r_{i j \alpha}\right),
$$

where $F_{i j \alpha}$ is the $\alpha$-component of the force $\vec{F}_{i j}$ on particle $i$ arising from an overlap with particle $j$, $r_{i j \beta}$ is the $\beta$-component of the vector $\vec{r}_{i j}$ from the center of mass of particle $j$ to that of particle $i$.

The correct form for the stress tensor $\hat{\Sigma}_{\alpha \beta}$ in $2 D$ for systems composed of anisotropic particles is the Love expression [16]:

$$
\hat{\Sigma}_{\alpha \beta}^{L}=\frac{1}{2 L^{2}} \sum_{i, j=1}^{N}\left(F_{i j \alpha} p_{i j \beta}+F_{i j \beta} p_{i j \alpha}\right),
$$

where $p_{i j \beta}$ is the $\beta$-component of the vector from the center of mass of particle $i$ to the point of contact $C_{i j}$ with particle $j$. Note that the Love expression reduces to the virial expression for spherical particles.

In our studies of simple shear, we focus on the off-diagonal component of the stress tensor $\Sigma_{x y}$. Calculating the point of contact at each shear strain is computationally expensive; we have therefore used the virial expression $\Sigma_{x y}^{V}$ instead of the Love expression $\Sigma_{x y}^{L}$ to quantify the shear stress for both dimer and ellipse packings. As a check, we measured both $\Sigma_{x y}^{L}$ and $\Sigma_{x y}^{V}$ for dimers as a function of aspect ratio and compression. Fig. 13 shows that they give quantitatively similar results for $\alpha=1.1$ and $\alpha=1.5$ (for $\gamma<0.2$ ), and qualitatively similar results for $\alpha=1.5$ at large strain. In particular, the plastic response of dimer packings at small compressions is unaffected by the choice of the definition of the shear stress. 


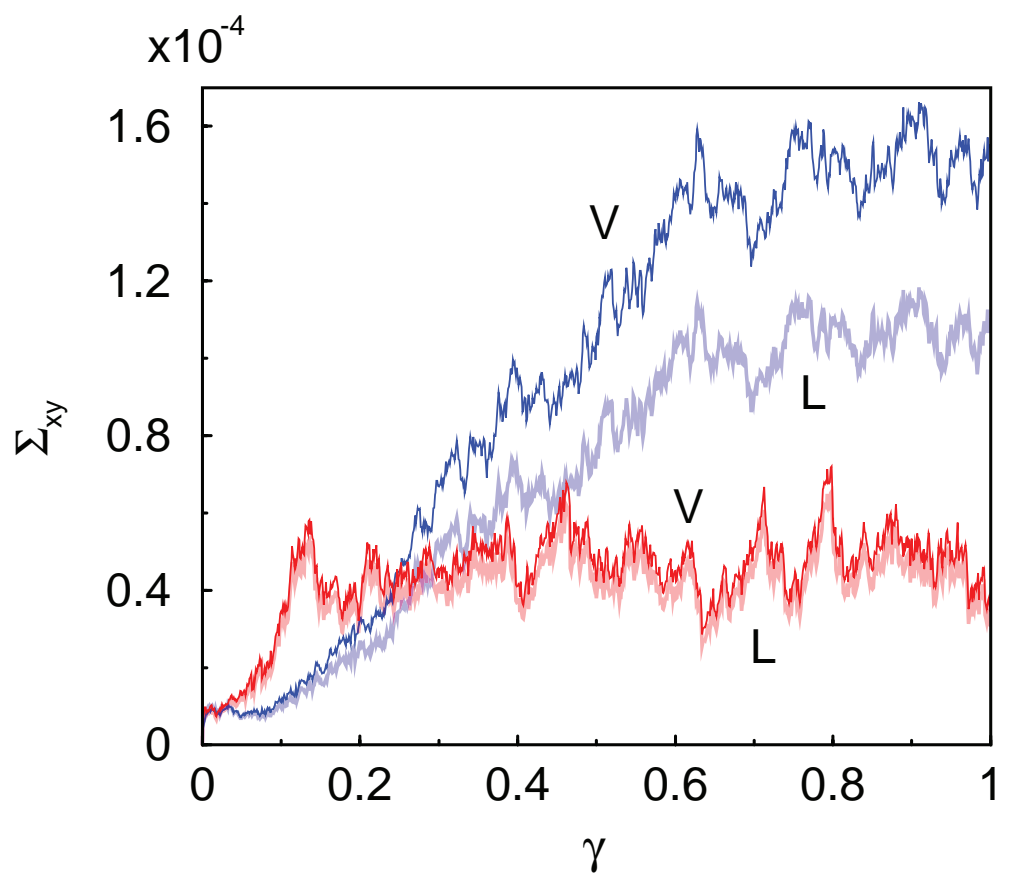

Figure 13: Comparison of the Love (L) and virial (V) expressions for the shear stress $\Sigma_{x y}$ as a function of shear strain $\gamma$ for aspect ratio $\alpha=1.1$ (red) and 1.5 (blue) at $\phi-\phi_{J}=10^{-3}$.

\section{References}

[1] A. J. Liu and S. R. Nagel, "Jamming is not just cool anymore," Nature 396, 21 (1998).

[2] Z. Zhang, N. Xu, D. N. Chen, P. Yunker, A. M. Alsayed, K. B. Aptowicz, P. Habdas, A. J. Liu, S. R. Nagel, and A. G. Yodh, "Thermal vestige of the zero-temperature jamming transition," Nature, 459, 230 (2009).

[3] M. van Hecke, "Jamming of soft particles: geometry, mechanics, scaling and isostaticity," $J$. Phys.: Condens. Matter 22, 033101 (2010).

[4] C. O. O'Hern, L. E. Silbert, A. J, Liu, and S. R. Nagel, "Jamming at Zero Temperature and Zero Applied Stress: The Epitome of Disorder", Phys. Rev. Lett. 68, 011306 (2003).

[5] W. G. Ellenbroek, E. Somfai, M. van Hecke, W. van Saarloos, "Critical Scaling in Linear Response of Frictionless Granular Packings near Jamming", Phys. Rev. Lett. 97, 258001 (2006).

[6] L. E. Silbert and A. J. Liu and S. R. Nagel, "Vibrations and diverging length scales near the unjamming transition," Phys. Rev. Lett. 95, 098301 (2005).

[7] W. G. Ellenbroek, M. van Hecke, and W. van Saarloos, "Jammed frictionless discs: Connecting local and global response," Physical Review E 80, 061307 (2009).

[8] M. Wyart and S. R. Nagel and T. A. Witten, "Geometric origin of excess low-frequency vibrational modes in weakly connected amorphous solids," Europhys. Lett. 72486 (2005).

[9] A. V. Tkachenko and T. A. Witten, "Stress propagation through frictionless granular materials," Phys. Rev. E 60, 687 (1999). 
[10] K. Desmond and S. V. Franklin, "Jamming of 3D prolate granular materials," Physical Review E 73, 031306 (2006).

[11] N. Mori and R. Semura and K. Nakamura, "Simple shear flows of suspensions of Brownian ellipsoids interacting via the Gay-Berne potential," Molecular Crystals and Liquid Crystals 367, 445 (2001).

[12] A. Donev, R. Connelly, F. H. Stillinger, and S. Torquato, "Underconstrained jammed packings of hard ellipsoids," Phys. Rev. E 75, 051304 (2007).

[13] Z. Zeravcic, N. Xu, A. Liu, S. Nagel, W. van Saarloos, "Excitations of ellipsoid packings near jamming," Europhys. Lett. 87, 26001 (2009).

[14] A. Donev, I. Cisse, D. Sachs, E. A. Variano, F. H. Stillinger, R. Connelly, S. Torquato, and P. M. Chaikin, "Improving the density of jammed disordered packings using ellipsoids," Science 303, 990 (2004).

[15] M. Mailman, C. F. Schreck, C. O. O'Hern, and B. Chakraborty, "Jamming in systems composed of frictionless ellipse-shaped particles", Phys. Rev. Lett. 102, 255501 (2009).

[16] S. F. Edwards and D. V. Grinev, "Transmission of stress in granular materials as a problem of statistical mechanics," Physica A 302, 162 (2001).

[17] D. J. Cleaver, C. M. Care, M. P. Allen, M. P Neal, "Extension and generalization of the Gay-Berne potential", Phys. Rev. E 54, 559 (1996).

[18] J. W. Perram and M. S. Wertheim, "Statistical mechanics of hard ellipsoids I. Overlap algorithm and the contact function", J. Comp. Phys. 58, 409 (1985).

[19] J. W. Perram, J. Rasmussen, E. Prætgaard, "Ellipsoid contact potential: Theory and relation to overlap potentials," Phys. Rev. E. 54, 6565 (1996).

[20] C. F. Schreck and C. S. O'Hern, "Computational methods to study jammed systems," in Experimental and computational techniques in soft condensed matter physics, Edited by J. S. Olafsen (Cambridge University Press, London, 2010).

[21] G.-J. Gao, J. Blawzdziewicz, and C. S. O'Hern, "Studies of the frequency distribution of mechanically stable disk packings", Phys. Rev. E 74, 061304 (2006).

[22] G.-J. Gao and J. Blawzdziewicz and C. S. O'Hern, "Geometrical families of mechanically stable granular packings," Phys. Rev. E 80, 061303 (2009).

[23] W. H. Press, B. P. Flannery, S. A. Teukolsky, and W. T. Vetterling, Numerical Recipes in Fortran 77 (Cambridge University Press, New York, 1986).

[24] M. P. Allen and D. J. Tildesley, Computer Simulation of Liquids (Oxford University Press, New York, 1987).

[25] N. Xu and C. S. O'Hern, "Measurements of the yield stress in frictionless granular systems," Phys. Rev. E 73, 061303 (2006).

[26] L. E. Silbert, D. Ertas, G. S. Grest, T. C. Halsey, D. Levine, and S. J. Plimpton, "Granular flow down an inclined plane: Bagnold scaling and rheology," Phys. Rev. E 64, 051302 (2001). 
[27] C. De Michele and R. Schilling and F. Sciortino, "Dynamics of uniaxial hard ellipsoids," Phys. Rev. Lett. 98, 265702 (2007). 